\title{
Surgical management of movement disorders
}

\author{
J M N Enslin, BPhysT, MB ChB, FCNeurosurgery (SA), MMed (Neurosurg) \\ Division of Neurosurgery, Red Cross War Memorial Children's Hospital, and Constantiaberg Mediclinic, Cape Town, South Africa
}

Corresponding author: J M N Enslin (enslin@functionalneurosurgery.co.za)

\begin{abstract}
Movement disorders are usually treated by neurologists, and appropriately so. The first-line management of all conditions that are grouped together as movement disorders (e.g. Parkinson's disease, dystonia, essential tremor) is with medication and, in some, with rehabilitative strategies, such as occupational therapy, physiotherapy and even psychotherapy. In general, if these strategies fail or have undesirable consequences, surgery would become an option. Intramuscular injection of botulinum toxin is also very useful in the focal dystonias, such as writer's cramp, and in the occupation-specific dystonias, such as musician's dystonia or hairdresser's dystonia. The limiting factor is the total safe dosage allowed. One cannot inject every muscle involved in all four limbs in a patient with generalised dystonia. This has led to surgery being offered as therapy in certain cases where there have previously not been any alternatives. Surgery does not offer curative procedures for movement disorders, but long-term control with medication is possible, often with significant reduction or complete cessation of symptoms.
\end{abstract}

S Afr Med J 2016;106(9):854-857. DOI:10.7196/SAMJ.2016.v106i9.11355

Disability from movement disorders is often seen as par for the course in the ageing process. It is the price paid by those who are lucky enough to grow old. There are, however, also young patients who develop Parkinson's disease (PD) and dystonia and have to discontinue their profession owing to a severely disabling tremor or task-specific dystonia, such as writer's cramp or musician's dystonia. In this group of patients, especially, awareness about surgical options to alleviate disability caused by their movement disorder is of the utmost importance.

Surgical procedures performed for movement disorders are classically divided into two categories (Table 1).

\section{Pathophysiology of movement disorders}

To understand the principles behind the surgical management of movement disorders, we first need to review the basic physiology of movement. This is a complex topic and a detailed discussion is beyond the scope of this article, but a brief overview is given.

Human movement is the end result of a complex, orchestrated and masterfully executed interaction of sensory feedback, modulation and action. Human movement is planned in the frontal lobes and is strongly linked to the need for reward. At the most primitive level, one performs an action to achieve an award.

Four cerebral areas are involved in human movement:

- primary motor cortex: e.g. sending the message via the corticospinal tracts to a limb

- secondary motor cortex (supplementary motor area): planning of movement

Table 1. Possible surgical procedures for treating movement disorders

\begin{tabular}{ll}
\hline Lesioning procedures & Neuromodulation \\
\hline Stereotactic lesioning of basal ganglia and/or thalamic targets & Deep brain stimulation \\
Selective peripheral denervation of specific muscles & Intrathecal baclofen therapy
\end{tabular}

- prefrontal cortex: adding emotional influence to our movements

- cingulate gyrus.

There are also subcortical structures involved in movement:

- basal ganglia: thalamus, globus pallidus and subthalamic nucleus (STN)

- cerebellum: 'fine tuning' of all movement takes place here

- white matter tracts: corticospinal tract, reticulospinal tracts and afferent tracts, e.g. spinothalamic.

The basal ganglia and cerebellum form part of a complex circuit controlled by positive and negative feedback loops. ${ }^{[1]}$ There is a network of connectivity between the thalamic nulei, cerebellum, STN and globus pallidus, which modifies and refines our actions. Dopamine plays an important role as the main neurotransmitter substance in this network. The physiological effect of lesioning or deep brain stimulation (DBS) on these nuclei is still unknown, but the most likely explanation is that the damping effect of PD is alleviated by high-frequency stimulation of the STN, while the inverse happens in dystonia, where stimulation of the globus pallidus interna (GPi) dampens the abnormal tone and movements. Much research is being done worldwide, with some units using viral tractography mapping studies that show the interconnectedness of all the abovementioned nuclei in controlling movements.

\section{Parkinson's disease}

PD occurs more commonly in patients of 55 - 65 years of age and it is estimated that up to $2 \%$ of people $>60$ years old will develop the condition. ${ }^{[2]} \mathrm{PD}$ is characterised by the clinical triad of bradykinesia, resting tremor and postural instability. ${ }^{[3]}$ Some of the associated features are bradymemia (slowness of thoughts), bradyphrenia and gait abnormalities, such as festination (shuffling gait). ${ }^{[3]}$ Also note that PD has associated non-motor symptoms. These are mostly resistant to all surgical interventions and include cognitive impairment, mood disturbances, sleep disturbances and even autonomic disorders. ${ }^{[4]}$ These non-motor symptoms often cause most dysfunction in previously high-performing individuals with PD. Care and support should therefore not be solely fixated on the movements or tremors, but a holistic multidisciplinary approach is essential.

DBS of the STN was recommended for patients with PD by the American Academy of Neurologists in 2006. ${ }^{[5]}$ The GPi is also a therapeutic target, but is best reserved for patients with PD where rigidity is the main feature. ${ }^{\left[{ }^{[5]}\right.}$ Ideal candidates for DBS are those with pre-operative good motor response to levodopa therapy during their 'on period. ${ }^{\text {? }}$ DBS has been shown to reduce the 'off time' 
in patients and the need for medication, and improve dyskinesia and motor function. ${ }^{[4]}$ The ideal candidates for DBS are summarised in Table 2. The reason for waiting for 5 - 6 years of symptomatic PD before considering DBS, is to allow ample time for excluding differential diagnoses such as other neurodegenerative diseases; also, most patients develop motor fluctuations and dyskinesias only after this time period. ${ }^{[7]}$ There is, however, good evidence to support the early use of DBS in patients with motor fluctuations caused by PD. ${ }^{[8]}$ Patients experience good resolution of these effects and therefore must be referred for surgical treatment options early in the disease. It is important to note that there is no benefit with regard to the non-motor effects, and there is some concern related to suicide risk, which is slightly higher after surgery. This may be due to the motor disability being relieved, but not the mood disturbances. ${ }^{[9]}$

\section{Dystonia}

Dystonia forms part of the complex of disorders that present with abnormal movements. It is classified into focal and generalised dystonia and further into primary and secondary causes. The mainstay of focal dystonia is local intramuscular injection with botulinum toxin, which causes local paralysis of the injected muscle fibres. ${ }^{[10]}$ This can allow the patient with focal types of dystonia, such as musician's cramp or cervical torticollis, to have normalised movements of their hands or neck, depending on the pathology and the selected injection sites. Botulinum toxin is effective for 5-6 months, and usually requires repeated injections to control the disability. In long-standing cases of focal dystonia, such as spasmodic cervical torticollis, selective peripheral denervation of the sternocleidomastoid muscle, trapezius and splenius capitis muscles in the neck can lead to significant improvement in the torticollis (the Bertrand procedure).

In focal dystonia of the hand some authors report very good long-lasting effects with contralateral lesioning of the ventral oralis anterior nucleus of the thalamus. ${ }^{[1]}$ Taira, from Japan, ${ }^{[11]}$ is renowned for having the patient awake during the procedure, with his/her musical instrument in the theatre, and asks the patient to perform a musical piece that would elicit their dystonia, while he is performing the surgical procedure. By doing this he confirms the therapeutic effect and avoids any damage to the surrounding internal capsule. Stereotactic placement of a DBS electrode in these thalamic nuclei, or in the GPi, has a similar effect, with the added benefit of removal if there are any side-effects and the ability to modify the stimulation parameters.

\section{Table 2. Ideal PD patient for DBS}

Adequate brain imaging that excludes alternative diagnosis

No cognitive impairment and motivated patient and family

Realistic expectations regarding the potential outcome of DBS

Duration of $\mathrm{PD}>5$ years

Proof of dopamine responsiveness (at least 30\% improvement in motor score with dopamine)

Problematic dyskinesia and motor fluctuations despite optimal medical therapy

Disabling medication-resistant tremor

Good medical health

No atypical parkinsonism

$\leq 80$ years of age

Generalised dystonia of genetic cause (specifically DYT1 abnormality) is the most responsive to DBS. Patients with DYT1 dystonia do extremely well after DBS; consequently, it is currently the first-line therapy.

DBS is expensive, but if the cost v. benefit analysis is taken into account there is significant functional gain for the patient, with long-term financial savings with regard to hospitalisation and chronic medication for funders. There is also major functional improvement and freedom of movement with the surgical management of dystonia patients.

\section{Essential tremor}

Essential tremor is one of the most common movement disorders and affects up to $5 \%$ of the general population. ${ }^{[12]}$ It may affect people from all age groups (most commonly the elderly) and has a strong familial predilection, with an autosomal-dominant inheritance pattern. ${ }^{[12]}$ Essential tremor is most often of a postural nature and is absent at rest. There is a clear exaggeration of this tremor with anxiety and stress, relieved by alcohol use. ${ }^{[13]}$ Medical management forms the mainstay of treatment (beta-blockers, gabapentin and other anticonvulsants). ${ }^{[13]}$

Once functional ability and quality of life are severely impaired by the tremor and it is refractory to medical treatment, DBS of the ventral intermediate nucleus of the thalamus is an option. Lesioning of the same nucleus (thalamotomy) may also be considered, with the drawback that the procedure is not reversible and may be associated with permanent neurological deficits after surgery. Up to $90 \%$ of patients experience a significant reduction in the number of tremors in their upper limbs and in the severity of the tremors. ${ }^{[13]}$

All patients who are candidates for DBS or lesioning procedures to treat their movement disorder need to undergo some basic blood tests, including a coagulation profile. This will reduce the risk of potentially catastrophic haemorrhagic complications. Generally, patients undergo brain imaging, magnetic resonance imaging (MRI) being the most useful. This is used to merge the stereotactic imaging and to exclude cerebral lesions and severe cerebral atrophy, which may be contraindications to DBS. Imaging also excludes differential diagnoses, such Wilson's disease - a dystonic condition treatable with medication rather than surgery.

\section{Surgical workflow}

The procedure is performed in one of two ways by most centres. Two different techniques are used to confirm the placement of the electrode in the chosen target. Fig. 1 summarises the procedures. The most common technique uses micro-electrode recording (MER) to confirm placement of the electrode. With this technique, a very thin electrode monitors the electrical activity at an individual neuronal level as it is passed through the brain parenchyma. The STN and GPi have characteristic electrical waveforms that are readily recognised by experienced neurosurgeons and neurophysiologists. Accuracy is therefore confirmed on a physiological level and the best electrode position is chosen during the surgical procedure. The same electrode can be used for stimulation, thereby checking for side-effects and unplanned effects on nearby structures, such as the optic tract or the internal capsule. Repositioning is then possible, during which the patient needs to be awake. Fig. 2 is an intraoperative image of this procedure. It may be difficult to perform in the elderly and younger children with dystonia, where the abnormal movements are so violent that the patient cannot be kept still in the head frame. Another approach, and the one I prefer, is to perform the entire procedure under general anaesthesia. The patient has the stereotactic frame applied, a stereotactic MRI brain scan is performed, and the target planning is done while the patient is anaesthetised. Once the planning has been done, the frame is applied over the frame base (Fig. 3) and the procedure is performed. Two burr holes are made behind the hairline and the electrodes are placed directly on the selected target. After placement of both electrodes a stereotactic MRI scan is 


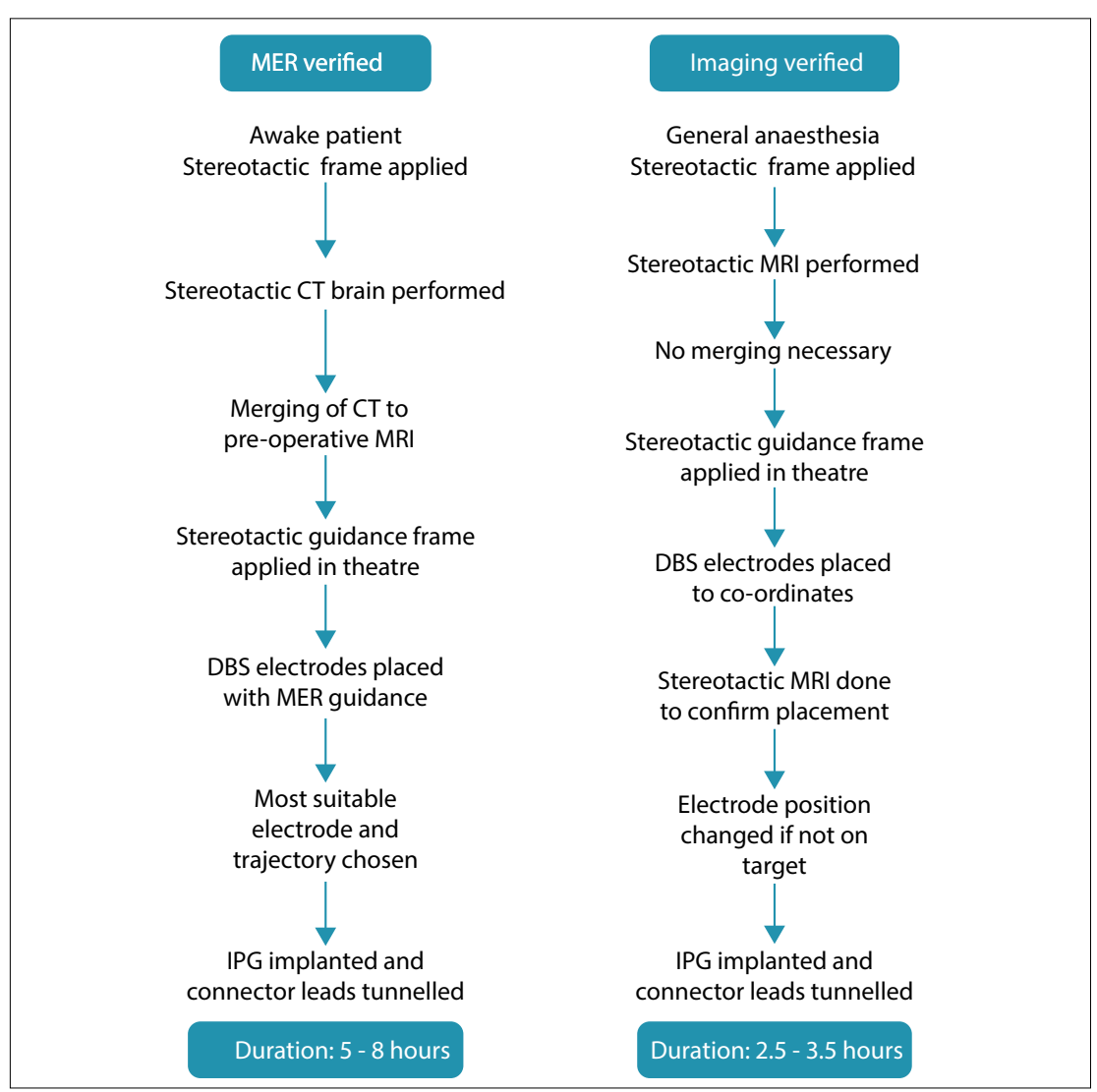

Fig. 1. Workflow of DBS summary, illustrating the difference between the two techniques $(M E R=$ micro-electrode recording; $C T=$ computed tomography; $M R I=$ magnetic resonance imaging; $I P G=$ implantable pulse generator).

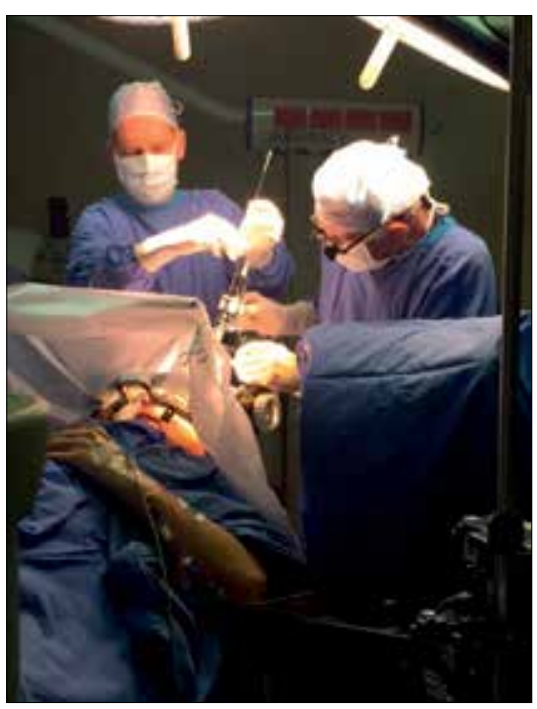

Fig. 2. The neurosurgical team placing the DBS electrode in a patient who is awake. The stereotactic frame is in situ on the patient's head.

performed again and imaging verification is done to confirm the position of the electrode in the chosen target (Fig. 4). If the placement is accepted, the implantable pulse generator (IPG) is inserted in theatre; if not, the electrode is first repositioned and then the procedure is completed. Frame-based stereotaxis is still the

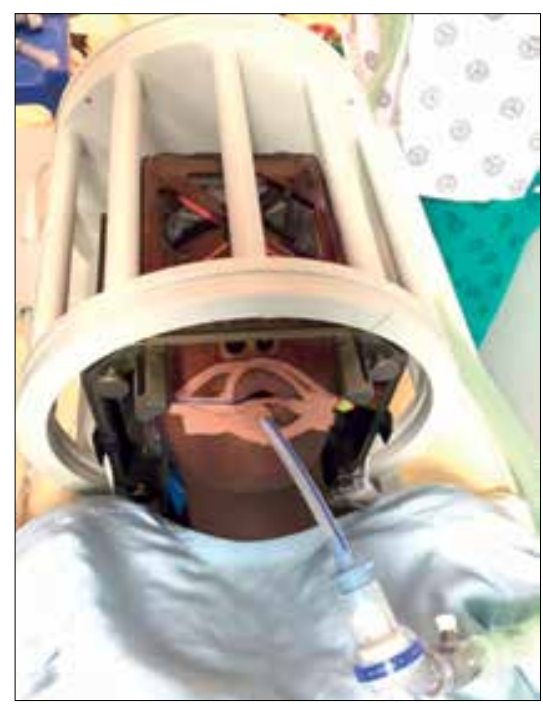

Fig. 3. A patient in the MRI head coil with stereotactic base and fiducial box on the head.

gold standard worldwide - the patient is fitted with a stereotactic frame that is bolted to the head - but there are some units that perform frameless stereotactic techniques.

The IPG is implanted in a subcutaneous pocket that is usually created on the left side of the chest wall. In very cachectic patients the IPG can be implanted in the submuscular plane to prevent skin breakdown over the device. The two electrodes are tunnelled under the skin to connect the implanted electrodes to the IPG - similar to a ventriculoperitoneal shunt placement.

The day after surgery the IPG is activated and a stepwise approach is used to test for side-effects and beneficial effects at a range of settings. The results of this initial screening procedure are used by the team to decide on the settings to be used when treating the patient.

Stereotactic lesioning techniques use the same workflow as described above, the only differences being that patients should not be operated on under anaesthesia and a radiofrequency electrode is placed stereotactically to the target in the basal ganglia. Once the target is reached, a stimulation pulse is used to check for side-effects and confirm the therapeutic effect. If there is no risk of damaging vital structures, such as the internal capsule, then a radiofrequency pulse is used to create a thermic lesion in the target nucleus. This leads to permanent disease modification by interrupting the intricate feedback mechanisms that have been discussed above.

\section{Follow-up}

Once the DBS procedure has been performed, follow-up of patients is the same as in those who do not qualify for DBS as treatment of their movement disorder. Patients tend to improve if they are kept on low doses of their medication, even if symptoms are well controlled without medication. In PD, for example, it is important to remember that DBS does not treat the non-motor effects of the disease, and the psychological and autonomic components are controlled by medication as before. However, the benefit is that dosages can be lowered to levels that cause fewer side-effects.

Two types of IPGs (pacemakers) are used: rechargeable and non-rechargeable. The advantage of the former is that it only needs replacement every $9-15$ years, depending on the manufacturer's specifications and the specific stimulation settings used (higher voltages at longer pulse width put more strain on the battery than inverse settings). The non-rechargeable implant will require replacement every 3 - 5 years, again depending on the settings used and the condition treated. Patient factors mostly determine which of the two types are used. Dependable caregivers or patients with a reliable electricity supply in their home will easily cope with the required once-weekly recharging session that is done with a magnetic recharging device that is placed, and kept, over the 


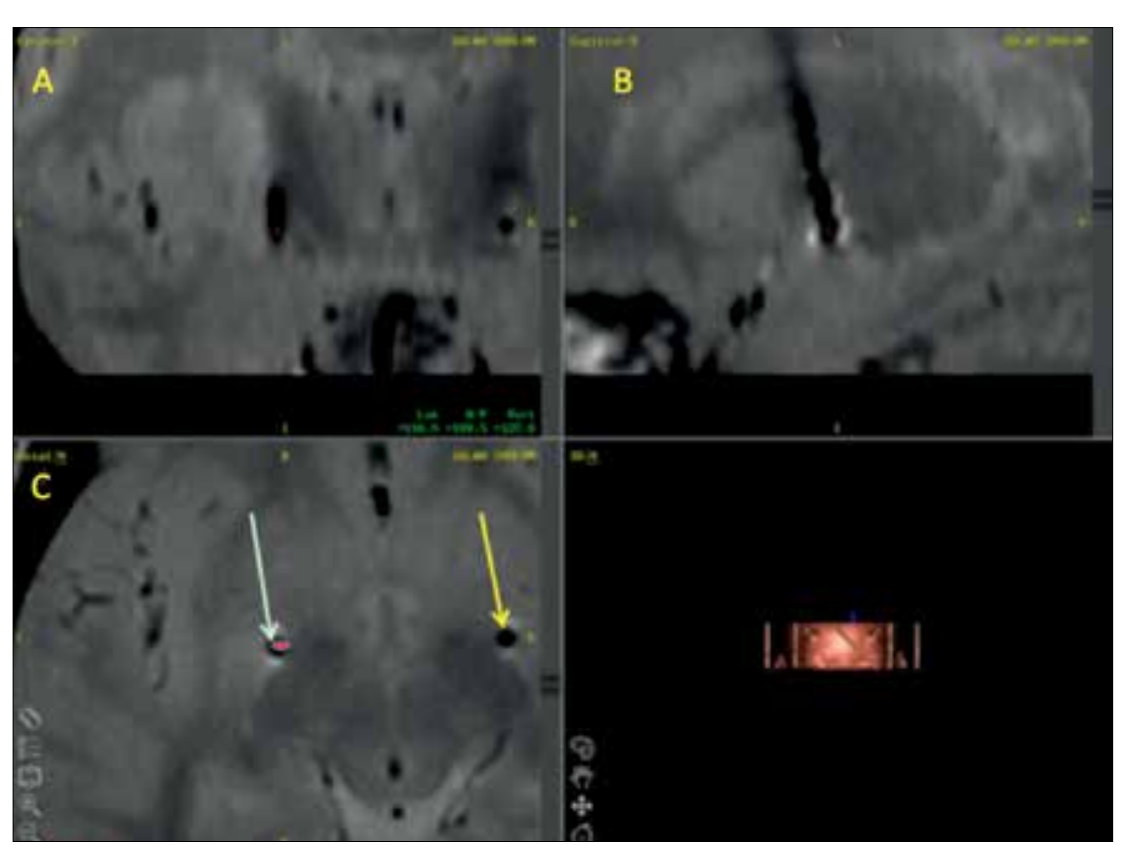

Fig. 4. Screenshot of a postoperative MRI scan showing the location of the electrode in the GPi bilaterally $((C)$ yellow arrow). The red dot $((C)$ green arrow) is the planned location that is superimposed on the postoperative image. This electrode is in the perfect location in the GPi. (A) Coronal section, (B) sagittal section, and $(C)$ axial section of DBS electrodes.

IPG for $\sim 2$ hours, allowing it to recharge. In doubtful circumstances or unreliable patients, the non-rechargeable option is fail safe. Changing of the IPG requires another operation, which is associated with higher wound infection rates than the primary surgery. This is the major reason for opting for a rechargeable device if financial and social circumstances allow.

DBS for dystonia differs from that for $\mathrm{PD}$ and essential tremor in one major way: the therapeutic effect of DBS in patients with dystonia has a slow onset, while in $\mathrm{PD}$ and essential tremor the effect is immediate. It is important that the patient with dystonia and their family realise this before the procedure, otherwise they are very disappointed and believe that the therapy was unsuccessful. Some units in Europe inform the patient that it will take months for the DBS to start working (personal experience), but in general there is at least some therapeutic effect in most patients in the first month after initiating the stimulation. Most patients experience a therapeutic effect within 6 months of the implantation. I prefer to switch on the stimulation device the day after surgery, but many surgeons rather wait a few days.
The rationale behind the waiting period is to allow for the slight swelling that is caused by the small electrode in the GPi or the STN to dissipate so that the effect of stimulation can be evaluated in isolation, without the lesioning effect playing a role in patient improvement.

The effect of the lesioning procedures, such as pallidotomy (lesioning in the globus pallidus) or subthalamic nucleotomy (lesioning in the STN), is immediate and permanent in most instances. However, even in these procedures, the therapeutic effect of globus pallidus lesioning will take time to manifest.

\section{Summary}

Surgical management of movement disorders becomes an option only when appropriate medical management has been exhausted and the patient's functioning and quality of life are impaired because of the disease. The abovementioned three diseases form the bulk of movement disorders treated by neurosurgeons, but currently targets for DBS are increasing by the month. Modern functional neurosurgeons treat pain conditions (e.g. cluster headaches) and psychiatric diseases (e.g. anorexia nervosa, major depres- sive disorder and obsessive compulsive disorder) with DBS and lesioning techniques. Certain conditions, especially psychiatric diseases where the cingulate gyrus is the target, lend themselves to lesioning rather than DBS. Therefore, lesioning techniques that have been performed since the mid1900 s still play an important role in modern neurosurgery.

It is generally agreed that surgery for movement disorders must only be performed in specific centres, where a multidisciplinary team is involved in the pre-operative work-up and the postoperative management of the patient. Neurosurgeons with special skills and aptitude for this type of work are needed; therefore, there are not many units in South Africa that offer this specialised service. Early referral is needed and more needs to be done to inform patients, their families and the medical fraternity of the surgical options for treating movement disorders.

\footnotetext{
1. Bajwa JA, Johnson MD, Vitek JL. Pathophysiology of dystonia. In: Lozano AM, Gildenberg PL, Tasker RR, eds. Textbook of Stereotactic and Functional Neurosurgery. Heidelberg: Springer, 2009:1779-1801.

2. Tanner CM, Aston DA. Epidemiology of Parkinson's disease

Tanner CM, Aston DA. Epidemiology of Parkinson's disease
and akinetic syndromes. Curr Opin Neurol 2000;13(4):427-430. and akinetic syndromes. Curr Opin Neurol

3. Lang AE, Lozano AM. Parkinson's disease. N Engl J Med 1998; 339(15):1044-1053. DOI:10.1056/nejm199810083391506

4. Chaudhuri KR, Martinez-Martin P, Schapira AH, et al. International multicenter pilot study of the first comprehensive self-completed nonmotor symptoms questionnaire for Parkinson's disease: The NMSQuest study. Move Disord 2006;21(7):916-923. DOI:10.1002/mds.20844

5. Pahwa R, Factor SA, Lyons KE, et al. Practice parameter: Treatment of Parkinson disease with motor fluctuations and dyskinesia (an evidence-based review): Report of the Quality Standards Subcommittee of the American the Quality Standards Subcommittee of the American Academy of
Neurology. Neurology 2006;66(7):983-995. DOI:10.1212/01. Neurology. Neurology 2006
wnl.0000215250.82576.87

6. Kazumata K, Antonini A, Dhawan V, et al. Preoperative indicators of clinical outcome following stereotaxic pallidotomy.
interative indicators of clinical outcome following stereotaxic pallidotomy.
Neurology 1997;49(4):1083-1090. DOI:10.1212/wnl.49.4.1083

Neurology 1997;49(4):1083-1090. DOI:10.1212/wnl.49.4.1083
7. Tan EK, Jancovic J. Patient selection for surgery for Parkinson's Tan EK, Jancovic J. Patient selection for surgery for Parkinson's
disease. In: Lozano AM, Gildenberg PL, Tasker RR, eds. Textbook of Stereotactic and Functional Neurosurgery. Heidelberg: Springer, 2009:1529-1539.

8. Schuepbach WMM, Rau J, Knudson K, et al. Neurostimulation for Parkinson's disease with early motor complications. N Engl J Med 2013;368(7):610-622. DOI:10.1056/NEJMoa1205158

9. Benabid AL, Mitrofanis J, Chabardes S, et al. Subthalamic nucleus stimulation for Parkinson's disease. In: Lozano AM, Gildenberg PL, Tasker RR, eds. Textbook of Stereotactic and Functional Neurosurgery. Heidelberg: Springer, 2009:1603-1631.

10. Persaud A, Garas G, Silva S, et al. An evidence-based review of botulinum toxin (Botox) applications in non-cosmetic
of of botulinum toxin (Botox) applications in non-cosmetic
head and neck conditions. JRSM Short Report 2013;4(2):1-9. head and neck conditions. JRSM

11. Taira T, Hori T. Stereotactic ventrooralis thalamotomy for taskspecific focal hand dystonia (writer's cramp). Stereotact Funct Neurosurg 2003;80(1-4):88-91. DOI:10.1159/000075165

12. Louis ED, Ottman R, Hauser WA. How common is the most common adult movement disorder? Estimates of the prevalence of essential tremor throughout the world. Move Disord 1998;13(1):5-10. DOI:10.1002/mds.870130105

13. Nazzaro JM, Lyons KE, Pahwa R. Management of essential tremor. In: Lozano AM, Gildenberg PL, Tasker RR, eds. Textbook of Stereotactic and Functional Neurosurgery. Heidelberg: Springer, 2009:1743-1757.
} 